\title{
Age-dependent decline in renal function in patients with lung cancer
}

\author{
GEN OHARA $^{1,2}$, KOICHI KURISHIMA ${ }^{2}$, KENSUKE NAKAZAWA ${ }^{2}$, MIO KAWAGUCHI $^{2}$, \\ KATSUNORI KAGOHASHI $^{1}$, HIROICHI ISHIKAWA ${ }^{3}$, NOBUYUKI HIZAWA ${ }^{2}$ and HIROAKI SATOH $^{1}$ \\ ${ }^{1}$ Division of Respiratory Medicine, Mito Medical Center, University of Tsukuba; ${ }^{2}$ Division of Respiratory Medicine, \\ Institute of Clinical Medicine, University of Tsukuba; ${ }^{3}$ Division of Respiratory Medicine, \\ Tsukuba Medical Center, Ibaraki, Japan
}

Received January 3, 2012; Accepted March 30, 2012

DOI: $10.3892 / \mathrm{ol} .2012 .672$

\begin{abstract}
The aim of this study was to clarify the effect of aging on renal function. Serum creatinine (SCr), blood urea nitrogen (BUN) and 24-h creatinine clearance (measured$\mathrm{CrCl}$ ) were examined in lung cancer patients and the measured- $\mathrm{CrCl}$ were compared with $\mathrm{CrCl}$ estimates by employing two commonly used equations. In total, 787 lung cancer patients who were diagnosed between 2001 and 2010 were retrospectively analyzed. SCr and urine creatinine, BUN and measured- $\mathrm{CrCl}$ were evaluated prior to treatment. The Cockcroft-Gault (CG) and modification of diet in renal disease (MDRD) formulae were also used to estimate $\mathrm{CrCl}$. $\mathrm{SCr}, \mathrm{BUN}$ and measured- $\mathrm{CrCl}$ showed a significant decline in the elderly. In the 787 lung cancer patients, a significant correlation coefficient was found between measured- $\mathrm{CrCl}$ and age. However, in patients aged 80 years or older, no significant correlation coefficient was found between measured- $\mathrm{CrCl}$ and age. In the comparison between the measured $\mathrm{CrCl}$ and the $\mathrm{CrCl}$ estimated by the two formulae, the $\mathrm{CG}-\mathrm{CrCl}$ levels were lower than those of the measured- $\mathrm{CrCl}$, whereas the MDRD$\mathrm{CrCl}$ levels were higher. Age is a crucial factor influencing renal function in patients with lung cancer. Particularly in the elderly, a decline in $\mathrm{CrCl}$ and greater individual variability in $\mathrm{CrCl}$, as well as discrepancies in measured- $\mathrm{CrCl}$ and estimated $\mathrm{CrCls}$ are significant factors.
\end{abstract}

\section{Introduction}

Lung cancer in the elderly is an increasingly common problem faced by the oncologist. Elderly patients have more co-morbid diseases and tend to tolerate toxic medical treatments more poorly than their younger counterparts. Recently, however,

Correspondence to: Dr Hiroaki Satoh, Division of Respiratory Medicine, Mito Medical Center, University of Tsukuba, Miya-machi 3-2-7, Mito, Ibaraki 310-0015, Japan

E-mail: hirosato@md.tsukuba.ac.jp

Key words: renal function, creatinine clearance, lung cancer, elderly clinical trials of platinum-based chemotherapy for selected elderly patients with good condition have been conducted, attracting attention to the utility of such therapy for the elderly $(1,2)$. Assessment of renal function is a daily clinical task, particularly in geriatric practice. Impairment of renal function is common in elderly patients, and their glomerular filtration rate (GFR) should be considered prior to prescription of drugs that are eliminated through the renal route or performing investigations requiring iodine injection. In clinical practice, GFR is rarely measured, since it is expensive and requires the use of radioelements for isotopic clearance determination, therefore renal function is assessed by the determination of creatinine clearance $(\mathrm{CrCl})$. Measured- $\mathrm{CrCl}$ has been used as the reference method since direct measurement of GFR using isotopic clearance is difficult to perform in elderly patients, and it is recognized to be a reliable estimate of GFR in older individuals (3). Nevertheless, the number of lung cancer patients older than 80 years evaluated for $\mathrm{CrCl}$ in previous studies has been too small $(4,5)$. Therefore, additional studies focusing especially on renal function in patients aged over 80 years are required. To clarify the effect of aging on renal function, we measured serum creatinine ( $\mathrm{SCr}$ ), blood urea nitrogen (BUN), and 24-h creatinine clearance (measured- $\mathrm{CrCl}$ ) in elderly patients with lung cancer and compared the measured- $\mathrm{CrCl}$ with $\mathrm{CrCl}$ estimates obtained from two commonly used formulae for $\mathrm{CrCl}$.

\section{Patients and methods}

Patients. Data from 787 patients with newly diagnosed lung cancer who were admitted to the Division of Respiratory Medicine, Tsukuba University Hospital or Tsukuba Medical Center Hospital between January 2001 and April 2010 were retrospectively analyzed. For each patient, the diagnosis of lung cancer was confirmed with pathological and/or cytological specimens. Pathological and/or cytological diagnosis was defined by the WHO classification and patients were staged according to the seventh edition of the cancer staging system (6). Age, height, actual body weight and gender were recorded at the initial visit. The evaluation of clinical data for lung cancer patients was approved by the institutional ethics committee of University of Tsukuba Hospital. 
Table I. Characteristics of 787 patients with lung cancer.

Characteristic

\begin{tabular}{lr}
\hline Age (years) & Median: 70, range: $21-87$ \\
$\leq 49$ & $30(3.8 \%)$ \\
$50-59$ & $121(15.4 \%)$ \\
$60-69$ & $251(31.9 \%)$ \\
$70-79$ & $327(41.6 \%)$ \\
$\geq 80$ & $58(7.4 \%)$ \\
Gender & \\
Male & $631(80.2 \%)$ \\
Female & $156(19.8 \%)$ \\
Histology & \\
Adenocarcinoma & $374(47.5 \%)$ \\
Squamous cell carcinoma & $191(24.3 \%)$ \\
Small cell carcinoma & $178(22.6 \%)$ \\
Large cell carcinoma & $20(2.5 \%)$ \\
Others & $24(3.0 \%)$ \\
Clinical stage & \\
IA-IIIA & $242(30.7 \%)$ \\
IIIB & $211(26.8 \%)$ \\
IV & $334(42.4 \%)$ \\
\hline
\end{tabular}

Creatinine clearance $(\mathrm{CrCl})$. Urinary and serum creatinine and BUN were measured using the enzymatic method. Prior to commencement of the lung cancer treatment, measured- $\mathrm{CrCl}$ in all patients was calculated three times and averaged. For each patient, the $\mathrm{CrCl}$ was also estimated using the Cockcroft and Gault (CG) and modification of diet in renal disease (MDRD) formulae as follows:

$$
\begin{gathered}
\mathrm{BSA}=0.007184 \times \mathrm{Xt}^{0.725} \times \mathrm{Wt}^{0.425} \\
\text { Measured-CrCl }=(1.73 / \mathrm{BSA}) \times(\mathrm{UCr} \times \mathrm{Uvol}) /(24 \times 60 \times \mathrm{SCr}) \\
\mathrm{CG}-\mathrm{CrCl}=(1.73 / \mathrm{BSA}) \times\{(140-\mathrm{A}) /
\end{gathered}
$$$$
\left.\left.(\mathrm{SCr}+0.2)^{*}\right\} \times(\mathrm{Wt} / 72) \times 0.85 \text { (inthecaseofafemalepatient }\right)(7)
$$

MDRD- $\mathrm{CrCl}=(1 / 0.719) \times 194 \times \mathrm{SCr}^{-1.094} \mathrm{x} \mathrm{A}^{-0.287} \times 0.739$

(in the case of a female patient) $(8,9)$

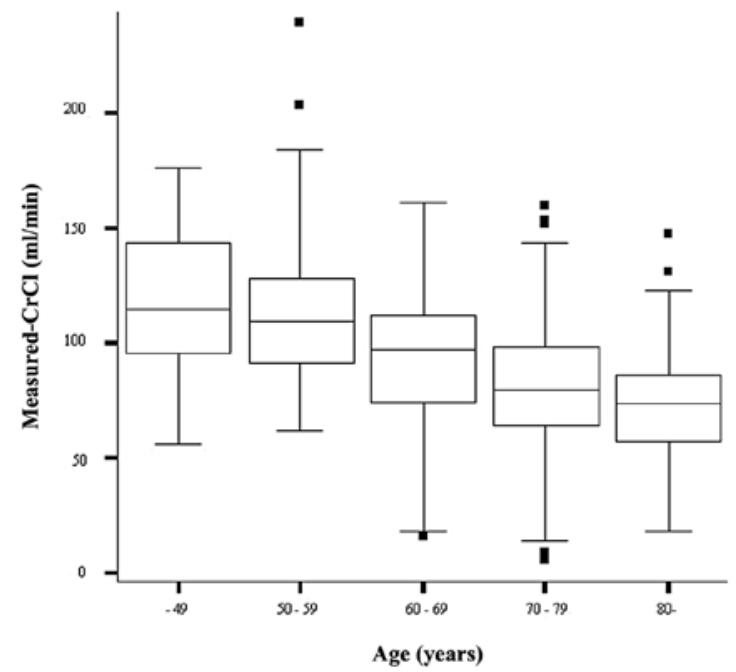

Figure 1. The measured- $\mathrm{CrCl}$ among the decades of age. The measured- $\mathrm{CrCl}$ decreased in an age-dependent manner, with significant differences among the age decades ( $\mathrm{P}=0.001$, Kruskal-Wallis test).

where BSA indicates body surface area $\left(\mathrm{m}^{2}\right)$; Ht, height $(\mathrm{cm})$; $\mathrm{Wt}$, body weight $(\mathrm{kg})$; UCr, urine creatinine concentration $(\mathrm{mg} /$ dl) (using the enzymatic method); Uvol, $24 \mathrm{~h}$ urine volume (ml/day); SCr, serum creatinine concentration ( $\mathrm{mg} / \mathrm{dl}$ ) (using the enzymatic method); and A, age (years). We added 0.2 to a $\mathrm{SCr}$ value measured using an enzymatic peroxidaseantiperoxidase method in order to render it equivalent to the SCr value measured using the Jaffē method (10).

Statistical analysis. The Mann-Whitney U test or KruskalWallis test was applied to elucidate the difference between two or more independent groups. Statistical significance between paired baseline and $\mathrm{CrCl}$ levels was evaluated using the Wilcoxon signed-rank test. To evaluate the correlation between two independent groups, the Spearman's rank correlation was used. Statistical analysis were performed using SPSS 10.1 for Windows (SPSS, Chicago, IL, USA). P $<0.05$ was considered statistically significant.

\section{Results}

Patient characteristics. Of the 787 patients included in the

\begin{tabular}{|c|c|c|c|c|}
\hline Age (years) & $\begin{array}{l}\text { Creatinine (mg/dl) } \\
\text { (median, range) }\end{array}$ & P-value ${ }^{a}$ & $\begin{array}{c}\text { BUN (mg/dl) } \\
\text { (median, range) }\end{array}$ & P-value ${ }^{a}$ \\
\hline$\leq 49$ & $0.60,0.37-1.10$ & \multirow[t]{5}{*}{0.001} & $11.7,5.5-19.8$ & \multirow[t]{5}{*}{0.001} \\
\hline $50-59$ & $0.70,0.39-1.19$ & & $13.4,5.3-27.1$ & \\
\hline $60-69$ & $0.70,0.30-2.20$ & & $14.9,5.3-44.9$ & \\
\hline $70-79$ & $0.80,0.20-5.40$ & & $16.0,3.7-86.4$ & \\
\hline$\geq 80$ & $0.80,0.79-2.60$ & & $17.1,5.6-53.9$ & \\
\hline
\end{tabular}
present study, $80.2 \%(\mathrm{n}=631)$ were men (Table $\mathrm{I})$. The median

Table II. Serum levels of creatinine and blood urea nitrogen in each age decade.

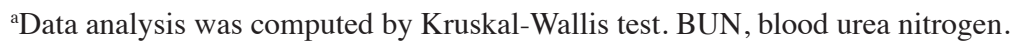


Table III. Value of measured-CrCl, CG-CrCl and MDRD-CrCl.

\begin{tabular}{lccc}
\hline Patient age (years) & $\begin{array}{c}\text { Measured } \mathrm{CrCl} \\
\text { (median, range) }\end{array}$ & $\begin{array}{c}\text { CG-CrCl } \\
\text { (median, range) }\end{array}$ & $\begin{array}{c}\text { MDRD-CrCl } \\
\text { (median, range) }\end{array}$ \\
\hline All patients & $89.4,5.1-239.0$ & $63.1,6.3-141.2^{\mathrm{a}}$ & $104.4,8.3-339.9^{\mathrm{a}}$ \\
$<70$ years & $102.1,15.8-239.3$ & $72.4,22.5-141.2^{\mathrm{a}}$ & $114.1,27.5-228.7^{\mathrm{a}}$ \\
$70-79$ years & $79.7,5.1-159.3$ & $54.9,6.3-124.4^{\mathrm{a}}$ & $99.8,8.3-339.9^{\mathrm{a}}$ \\
$\geq 80$ years & $72.6,17.9-147.2$ & $45.1,17.2-71.8^{\mathrm{a}}$ & $85.2,27.0-163.4^{\mathrm{a}}$ \\
\hline
\end{tabular}

$\mathrm{CrCl}$, Creatinine clearance; $\mathrm{CG}$, Cockcroft-Gault; MDRD, modification diet in renal disease. ${ }^{\mathrm{a}} \mathrm{P}=0.001$ versus measured-CrCl.

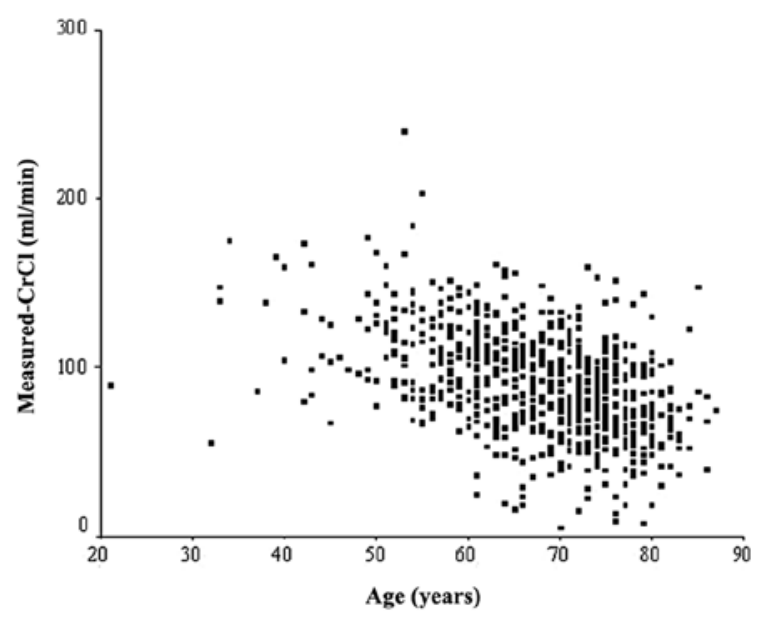

Figure 2. Correlation between measured- $\mathrm{CrCl}$ and age in all 787 lung cancer patients. A statistically significant correlation coefficient was found between measured- $\mathrm{CrCl}$ and age (Spearman's p-value $=-0.447, \mathrm{P}=0.001)$.

age was 70 years (range, 21-87). The types of lung cancer comprised 609 non-small cell carcinomas and 178 small cell carcinomas. Stage IA-IIIA was noted in 242 patients, stage III-B in 211 patients, and stage IV disease in 334 patients.

Renal function with age. SCr and BUN levels were determined in each decade of age (Table II). Both Scr and BUN showed a statistically significant difference among the age decades ( $\mathrm{P}=0.001$, Kruskal-Wallis test). The measured-CrCls decreased in an age-dependent manner, with significant differences among the age decades $(\mathrm{P}=0.001$, Kruskal-Wallis test $)$ (Fig. 1). The $\mathrm{CG}-\mathrm{CrCl}$ and $\mathrm{MDRD}-\mathrm{CrCl}$ also decreased in an age-dependent manner $(\mathrm{P}=0.001$ and $\mathrm{P}=0.001$, respectively, data not shown).

Correlation between $\mathrm{CrCl}$ and age. In all the 787 lung cancer patients, a statistically significant correlation coefficient was found between measured- $\mathrm{CrCl}$ and age (Spearman's p-value $=-0.447, \mathrm{P}=0.001)$ (Fig. 2). In patients aged $\geq 70$ years, a statistically significant correlation coefficient was also found between measured-CrCl and age, although Spearman's p-value was extremely low (Spearman's p-value $=-0.172, \mathrm{P}=0.001$ ) (Fig. 3). However, in patients aged $\geq 80$ years, a statistically significant correlation coefficient was not found (Spearman's $\mathrm{p}$-value $=-0.100, \mathrm{P}=0.456$, data not shown).

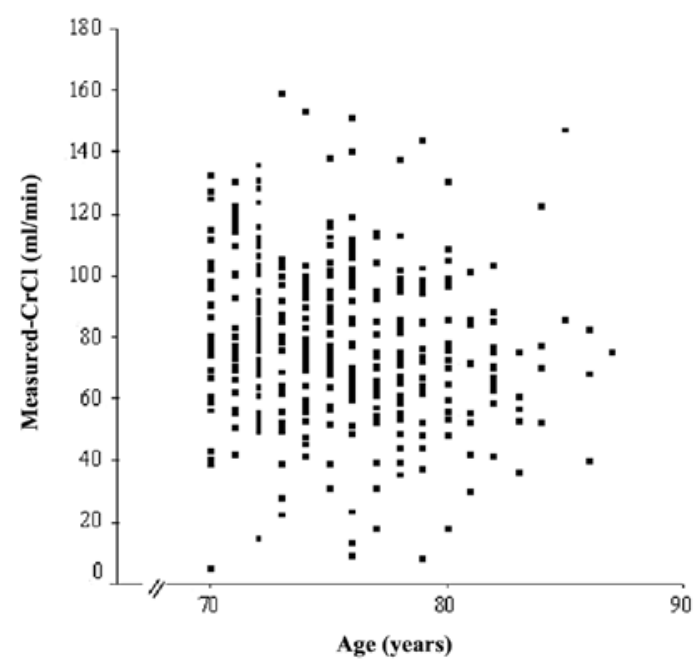

Figure 3. Correlation between measured- $\mathrm{CrCl}$ and age in patients aged $\geq 70$ years. A statistically significant correlation coefficient was also found between measured- $\mathrm{CrCl}$ and age, although the Spearman's p-value was extremely low (Spearman's p-value $=-0.172, \mathrm{P}=0.001$ ).

Comparison among measured-CrCl, CG-CrCl and MDRD$\mathrm{CrCl}$. The median values and ranges of the measured- $\mathrm{CrCl}$, CG-CrCl, and MDRD-CrCl are shown in Table III. In all 787 patients, the $\mathrm{CG}-\mathrm{CrCl}$ levels were significantly lower than those of the measured- $\mathrm{CrCl}(\mathrm{P}=0.001$, Wilcoxon signed-rank test). However, the MDRD-CrCl levels were significantly higher than those of the measured- $\mathrm{CrCl}(\mathrm{P}=0.001)$. Particularly in patients aged 70-79 years, the $\mathrm{CG}-\mathrm{CrCl}$ levels were lower than the measured-CrCl levels, and the MDRD-CrCl levels were significantly higher than the measured- $\mathrm{CrCl}$ levels ( $\mathrm{P}=0.001$ and $\mathrm{P}=0.001$, respectively). These discrepancies were also observed in patients aged $\geq 80$ years.

\section{Discussion}

With recent improvements in the medical management of lung cancer, the availability of many new drugs, better standards of medical care and more widespread health services, survival of elderly lung cancer patients has improved. In addition, Quoix et al recently reported that treatment with platinumbased doublet chemotherapy resulted in better survival for the very elderly with non-small cell lung cancer than did standard single-agent therapy (11). As the proportion of elderly patients 
aged 80 years or older continues to increase, intensive chemotherapy is likely to bring a clinical benefit to elderly patients with clinically good conditions such as unimpaired renal function. However, few studies evaluating renal function in a large number of elderly patients with lung cancer have been published thus far.

Renal function is known to decrease with age and morphological changes, such as decrease of kidney weight, appearance of sclerotic glomeruli (12) and intimal proliferation in the renal artery, are some of the causes of renal dysfunction (13). $\mathrm{SCr}$ concentration remains the most widely used index of renal function in clinical practice (14). In the very elderly, however, serum creatinine is not always useful as a marker of renal function since creatinine production is low due to decreased muscle mass associated with aging $(15,16)$. The GFR is generally used as an index of renal function and may be accurately measured through the renal clearance of either cold (inulin, iohexol) or radiolabeled (51Cr-EDTA, 99mTc-DTPA) exogenous filtration markers. Nonetheless, these methods are seldom used in clinical practice as they are invasive and expensive and require the use of radioelements for isotopic clearance determination. Instead, indirect methods are used for bedside renal function estimates, all of which are based on $\mathrm{CrCl}$.

In oncological practice, estimation of GFR at the bedside is essential since renal insufficiency is directly associated with increased chemotherapeutic complications. The two most commonly used formulae, the CG and MDRD, were developed to estimate the $\mathrm{CrCl}(7,17,18)$. However, it is notable that in the original report in which the $\mathrm{CG}$ equation was validated, only 17 of a total of 249 patients were aged over 80 years (7). In addition, the MDRD formula was developed to estimate $\mathrm{CrCl}$ in patients with a variety of chronic renal diseases (19) and this population differs from those recruited for $\mathrm{CrCl}$ estimation with the $\mathrm{CG}$ formula as well as from elderly cancer patients such as those of the present study. Therefore, these two commonly used equations have not yet established an optimal accuracy in very elderly patients with lung cancer. Furthermore, a marked discrepancy between the CG and the MDRD formulae has been observed in a large population of over 65 years of age (20) and these formulae have been found to lack a consistent proportion of patients with renal failure within a population of octogenarian inpatients (21). Studies estimating renal function in solid cancer patients have previously been published (22-25). However, the number of the cancer patients aged 80 years or older evaluated for renal function was limited $(24,25)$. Therefore, additional studies focusing specifically on renal function in very elderly lung cancer patients are required. To clarify the effect of aging on renal function, we evaluated SCr, BUN, and measured- $\mathrm{CrCl}$ in elderly patients with lung cancer. SCr concentration and BUN showed statistically significant increase among the decades of age, whereas measured $\mathrm{CrCl}$ showed a statistically significant decrease among the age decades. Analysis of the correlation between measured$\mathrm{CrCl}$ and age showed a statistically significant correlation coefficient in all 787 lung cancer patients. A significant but weaker correlation coefficient was observed in patients aged 70 years or older. In patients aged 80 years or older, however, a statistically significant correlation coefficient was not found between measured- $\mathrm{CrCl}$ and age. We also compared the measured-CrCl, CG- $\mathrm{CrCl}$ and MDRD-CrCl levels. In all 787 patients, the $\mathrm{CG}-\mathrm{CrCl}$ levels were significantly lower than those of the measured- $\mathrm{CrCl}$, whereas the MDRD- $\mathrm{CrCl}$ levels were significantly higher. Particularly in patients aged 70 and over, $\mathrm{CG}-\mathrm{CrCl}$ was lower than the measured- $\mathrm{CrCl}$, and the MDRD- $\mathrm{CrCl}$ levels were significantly higher than those of the measured-CrCl. Our results are consistent with those obtained by Péquignot et al from 121 patients aged 70 and older with an indwelling urinary catheter (4).

Despite the novel findings of the present study, there are several limitations. Firstly, GFR was not determined using the isotopic reference method. Such methods are difficult to use in elderly patients with lung cancer as they are invasive and seldom readily available in oncological practice. The use of the isotopic method in the elderly patients of our study would have introduced a major selection bias. Secondly, this study was retrospective and our findings were obtained from a limited number of patients that were neither population-based nor case-controlled. Nevertheless, we suggest that it is of interest to report the management of unselected groups of elderly lung cancer patients. In the present study, we confirmed the importance of age as a factor influencing renal function in patients with lung cancer. As an early detection method and curative therapy have yet to be established, all lung cancer patients have a high possibility of receiving chemotherapy at the time of the first presentation or recurrence. Advanced lung cancer patients are particularly likely to receive chemotherapy. In the present study, we revealed a decline in $\mathrm{CrCl}$ in the elderly. In addition, we showed greater individual variability in $\mathrm{CrCl}$ in elderly lung cancer patients. For elderly patients, therefore, careful evaluation of renal function is essential to avoid excessive dosage of chemotherapeutic drugs.

\section{Acknowledgements}

We are especially grateful to Ms. F. Miyamasu for her review of the manuscript.

\section{References}

1. Vamvakas L, Saloustros E, Karampeazis A and Georgoulias V: Advanced non-small-cell lung cancer in the elderly. Clin Lung Cancer 10: 158-167, 2009.

2. Lang K, Marciniak MD, Faries D, et al: Trends and predictors of first-line chemotherapy use among elderly patients with advanced non-small cell lung cancer in the United States. Lung Cancer 63: 264-270, 2009.

3. Lamb EJ, Webb MC, Simpson DE, et al: Estimation of glomerular filtration rate in older patients with chronic renal insufficiency: is the modification of diet in renal disease formula an improvement? J Am Geriatr Soc 51: 1012-1017, 2003.

4. Péquignot R, Belmin J, Chauvelier S, et al: Renal function in older hospital patients is more accurately estimated using the Cockcroft-Gault formula than the modification diet in renal disease formula. J Am Geriatr Soc 57: 1638-1643, 2009.

5. Verhave JC, Fesler P, Ribstein J, et al: Estimation of renal function in subjects with normal serum creatinine levels: influence of age and body mass index. Am J Kidney Dis 46: 233-241, 2005.

6. Lababede O, Meziane M and Rice T: Seventh edition of the cancer staging manual and stage grouping of lung cancer: quick reference chart and diagrams. Chest 139: 183-189, 2011.

7. Cockcroft DW and Gault MH: Prediction of creatinine clearance from plasma creatinine. Nephron 16: 31-41, 1976.

8. Matsuo S, Imai E, Horio M, et al: Revised equations for estimated GFR from serum creatinine in Japan. Am J Kidney Dis 53: 982-992, 2009. 
9. Sato H, Kuroda T, Tanabe N, et al: Cystatin C is a sensitive marker for detecting a reduced glomerular filtration rate when assessing chronic kidney disease in patients with rheumatoid arthritis and secondary amyloidosis. Scand J Rheumatol 39: 33-37, 2010.

10. Horio $M$ and Orita Y: Comparison of Jaffē rate assay and enzymatic method for the measurement of creatinine clearance. Jap J Nephrol 38: 296-299, 1996.

11. Quoix E, Zalcman G, Oster JP, et al: Carboplatin and weekly paclitaxel doublet chemotherapy compared with monotherapy in elderly patients with advanced non-small-cell lung cancer: IFCT-0501 randomised, phase 3 trial. Lancet 378: 1079-1088, 2011.

12. Kaplan C, Pasternack B, Shah H, et al: Age-related incidence of sclerotic glomeruli in human kidneys. Am J Pathol 80: 227-234, 1975.

13. Tauchi H, Tsuboi K and Sato K: Histology and experimental pathology of senile atrophy of the kidney. Nagoya Med J 4: 71-97, 1958.

14. Levey AS: Measurement of renal function in chronic renal disease. Kidney Int 38: 167-184, 1990.

15. Beck LH: Changes in renal function with aging. Clin Geriatr Med 11: 199-209, 1998.

16. Herig PJ and Carlson RE: Plasma creatinine and renal function in the elderly. JAMA 248: 31, 1982.

17. Levey AS, Bosch JP, Lewis JB, et al: A more accurate method to estimate glomerular filtration rate from plasma creatinine: a new prediction equation. Modification of Diet in Renal Disease study group. Ann Intern Med 130: 461-470, 1999.
18. Levey AS, Greene T, Kusek JW, et al: A simplified equation to predict glomerular filtration rate from plasma creatinine. J Am Soc Nephrol 11: A0828, 2000.

19. National Kidney Foundation: K/DOQI clinical practice guidelines for chronic kidney disease: evaluation, classification, stratification. Am J Kidney Dis 39: S1-S266, 2002.

20. Garg AX, Papaioannou A, Ferko N, et al: Estimating the prevalence of renal insufficiency in seniors requiring long-term care. Kidney Int 65: 649-653, 2004.

21. Rimon E, Kagansky N, Cojocaru L, Gindin J, Schattner A and Levy S: Can creatinine clearance be accurately predicted by formulae in octogenarian in-patients? QJM 97: 281-287, 2004.

22. Nishimura G, Tsukuda M, Horiuchi C, et al: Decrease of creatinine clearance rate with aging in patients with head and neck cancer in Japan. Int J Clin Oncol 12: 120-124, 2007.

23. Hurria A, Hurria A, Brogan K, et al: Effect of creatinine clearance on patterns of toxicity in older patients receiving adjuvant chemotherapy for breast cancer. Drugs Aging 22: 785-791, 2005

24. Séronie-Vivien S, Toullec S, Malard L, et al: Contribution of the MDRD equation and of cystatin $\mathrm{C}$ for renal function estimates in cancer patients. Med Oncol 23: 63-73, 2006.

25. Thyss A, Saudes L, Otto J, et al: Renal tolerance of cisplatin in patients more than 80 years old. J Clin Oncol 12: 2121-2125, 1994. 